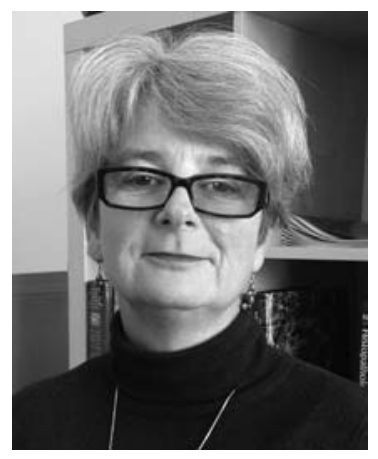

\title{
A Conversation with Gabriele Bergers
}

\author{
Interviewer: Gemma Alderton
}

Senior Editor, Nature Reviews Cancer

Gabriele Bergers is a Group Leader at the Flanders Institute for Biotechnology (VIB), a Professor in the Department of Onocology at KU Leuven, Belgium, and a Professor in the Department of Neurological Surgery at the University of California, San Francisco.

Gemma Alderton: You work on the tumor microenvironment and in particular on angiogenesis. Could you give us some background as to where we're at with that?

Dr. Bergers: We have realized that the old idea of depleting tumor vessels to starve a tumor to death, as Judah Folkman had proposed, is not working. It actually can make it worse sometimes, because hypoxic conditions can lead to recurrence and even a more aggressive growth. Rakesh Jain flipped the whole idea and came up with the concept of vessel normalization to make blood vessels more functional; this enables better drug delivery and therefore better efficacy. The problem is that it's a transient process. You have good efficacy for a while, but then further vessel pruning will lead again to hypoxia and tumor relapse. This is a big hurdle. Indeed, antiangiogenic therapies, specifically those targeting vascular endothelial growth factor (VEGF) receptor signaling pathways, show transient efficacies and only work in a subset of tumors. In the beginning, nobody really understood why, if you just block blood vessels, that it works in some tumors but it doesn't work in others. Several laboratories, including ours, started to identify some of the resistance mechanisms. These are very distinct and different because it's not just classic resistance where the cells don't respond to the inhibitor. Here, they find evasive programs and still grow in the presence of the inhibitor. With these issues came a new concept: Can we do combination therapy that targets these evasive pathways and thereby sustain antiangiogenic efficacy?

We approached two different resistance mechanisms. One entailed the implication of myeloid cells in resistance causing a proangiogenic relapse. The other mechanism was independent of the reactivation of the angiogenic program; rather, it was an alternative way to grow. In this scenario, tumors became more invasive - simply expanding by co-opting and crawling along existing blood vessels. This appears to happen in a subset of gliomas. It's not that glioblastomas are not invasive, but they invade by different means and can enhance such angiotropic migration if necessary. Again, however, it only happens in a subset of tumors, so why does GBM A do it and GBM B doesn't do it?

That's when we started investigating the underpinning mechanisms in more detail. We realized that there is not just one prototypic vascular niche in tumors; it's really a heterogeneous group of distinct niches. You have the typical angiogenic niches with sprouting, abnormal tumor vessels, and you can have hypoxic niches in which tumor vessels are nonfunctional or regressed, as well as invasive, co-opted tumor niches in which tumor cells move along normal blood vessels. Similarly, you can have metastatic niches where tumor cells extravasate and co-opt blood vessels in distant organs. We then asked, how does antiangiogenic therapy affect these distinct vascular niches?

We realized that the tumor niches responded very differently. It depended not only on the composition of the different tumor cells, but also on the composition of the host cells. There's a substantial diversity: The heterogeneous tumor cells and heterogeneous host cells interacting dynamically.

With regard to targeting the vascular niche, we are aware that anti-VEGF therapy is not efficient enough. The question is, is it still worthwhile to target the tumor vasculature? By studying combination of immune modulatory and antiangiogenic therapies we realized that not only can we make blood vessels more functional and create an immunestimulatory environment, but you can actually turn some of those into vascular structures reminiscent of high endothelial venules (HEVs): These are specialized blood vessels that are normally found in lymphoid organs to enable lymphocyte trafficking and seem to help here to get $\mathrm{T}$ cells into the tumor. We found for the first time that we can therapeutically induce HEV formation in some of these tumors, which correlated with a better tumor response.

Gemma Alderton: Is that because of clearance from the T cells?

(C) 2016 Bergers. This article is distributed under the terms of the Creative Commons Attribution-NonCommercial License, which permits reuse and redistribution, except for commercial purposes, provided that the original author and source are credited. 
Dr. Bergers: Yes, we think so. More T cells infiltrate and are activated. For many years the immunology field and the angiogenesis field have taken mostly separate paths. For example, we have been thinking about VEGF as a proangiogenic factor, but we were not thinking about it as an immune-suppressive factor. By blocking VEGF we can induce angiostatic as well as immunestimulatory effects. This comes back to the whole point that the immune system and the vasculature are functionally interregulated. During wounding, myeloid cells can become activated to attack and kill potential pathogens, but then they need to switch in the resolution phase to become immune-suppressive and angiogenic. It's a normal homeostasis program and the tumor takes advantage of this and can flip the cells to their own advantage. The good part is that you can flip the cells too, making them again angiostatic and immune stimulatory. This is an evolving theme in many laboratories. It will be important to see if one can induce these effects not only in primary tumors but also in metastatic lesions.

Tumor vessels themselves are highly immune-suppressive. They up-regulate PD-L1 (Programmed death ligand 1), for example, which serves as a negative checkpoint regulator on endothelial cells. If you have high PD-L1 levels on tumor vessels, and lower numbers of adhesion molecules, $\mathrm{T}$ cells do not sufficiently enter the tumor. That's the first barrier. These blood vessels become a gate. Instead of getting rid of tumor vessels, we need to turn them into vessels that can get lymphocytes into the tumor. Importantly though is to get the right cells in there and have them activated. This is where the reprogramming comes in. One needs to create an immunestimulatory environment with innate and adaptive immune cells.

There are a variety of clinical studies showing that spontaneous HEV formation in many human tumor types - breast cancer, melanomas, gastric cancer, colon cancer-mostly correlates with good prognosis and prolonged survival. The idea is, how can we use this therapeutically and target these blood vessels in a way that we can alter them and turn them into HEVs to enable more efficient T-cell infiltration in order to more efficiently attack the tumor.

Gemma Alderton: What are the real challenges in terms of your research? Is it modeling? What are the hurdles you need to overcome to move forward?

Dr. Bergers: It's always a problem when you start with mouse models and you need to show that it's really happening in patients. We would love to have tissue samples from patients who have been treated with these inhibitors to see if the hypothesis that we are trying to prove in mouse models really works in patients. This is often a major challenge because of the difficulties to obtain preand posttreated tumor samples from the same patient, or even just to obtain posttreated tumors because many patients will not undergo surgery anymore.

The other problem with antiangiogenic and immune modulatory therapy has been the lack of biomarkers. How do we know when response and relapse occurs? Many laboratories attempt to obtain signatures from peripheral blood of patients. We have looked into signatures of innate immune cells to test whether their changes within the tumor are also seen in those from peripheral blood. If one finds a signature, one could actually take blood from patients and oversee if the therapy is working and if, when it will stop working.

Gemma Alderton: That's challenging though, given the massively dynamic nature of those tumors.

Dr. Bergers: They're extremely dynamic, but that's what makes it interesting. 


\section{$\$_{\text {CSH\& }}^{\infty}$ Cold Spring Harbor Symposia SYMPOSIA}

\section{A Conversation with Gabriele Bergers}

Cold Spring Harb Symp Quant Biol 2016 81: 299-300 originally published online March 9, 2017 Access the most recent version at doi:10.1101/sqb.2016.81.031914

Creative This article is distributed under the terms of the

Commons http://creativecommons.org/licenses/by-nc/4.0/, which permits reuse and

License redistribution, except for commercial purposes, provided that the original author and source are credited.

Email Alerting Receive free email alerts when new articles cite this article - sign up in Service the box at the top right corner of the article or click here. 